\title{
Simple and cost-effective assay for isolating invasive living cells
}

\author{
Takahisa Takino*,1,2, Hiroshi Sato² \& Motoharu Seiki ${ }^{3}$ \\ ${ }^{1}$ Division of Education for Global Standard, Institute of Liberal Arts \& Science, Kanazawa University, \\ Kakuma-machi, Kanazawa 920-1192, Japan; 'Division of Molecular Virology \& Oncology, Cancer Research \\ Institute, Kanazawa University, Kakuma-machi, Kanazawa 920-1192, Japan; ${ }^{3}$ Faculty of Medicine, Institute \\ of Medical, Pharmaceutical \& Health Science, Kanazawa University, Takara-machi, Kanazawa 920-0934, \\ Japan
}

BioTechniques 65: 137-142 (September 2018) 10.2144/btn-2018-0036

Keywords: collagen gel $\bullet$ invasion assay $\bullet$ matrix metalloproteinase $\bullet$ three dimensional

A modified invasion assay using a three-dimensional collagen gel was developed that enables isolation of invasive living cells; it was named the invading cell trapping (iCT) assay. A small cell strainer consisting of a nylon mesh with 40- $\mathrm{m}^{2}$ pores was used, and collagen gel layers formed across the membrane. Test cells were seeded in the lower gel layer and invasive cells were attracted upward and trapped in the upper gel. After incubation, the collagen gel layers in cell strainers were easily separated and living cells in the gel were counted and analyzed. An advantage of the iCT assay is that it can capture living invasive cells in the upper gel while leaving noninvasive ones in the lower layer. Further enrichment of the two cell populations can be achieved by repeating the assay. Thus, the iCT assay allows comparative analysis of invasive versus noninvasive cells.

Cell invasion occurs during various physiological and pathological tissue remodeling processes, including during development and wound healing and in diseases related to tissue destruction, such as cancer. The extracellular matrix (ECM) is a complex network formed through structural and functional interactions of macromolecules that provides anchoring sites for cells, as well as signals that regulate cell proliferation, migration and differentiation [1,2]. It also acts as a barrier for cell movement in tissue; therefore, cell invasion usually requires the controlled destruction and restructuring of the ECM. Protein turnover in the ECM is triggered by proteolytic enzymes such as serine proteinases and matrix metalloproteinases (MMPs) that modulate tissue remodeling according to the type of cells [3-5]. Membrane type (MT)1-MMP was first identified as a tumor-specific MMP-2 activator, which stimulates tumor invasion and metastasis [6]. Later, multiple functions of MT1-MMP were demonstrated to be associated with tumor malignancy $[7,8]$.
For example, MT1-MMP degrades a wide range of ECM components including type I, II, III collagen, fibronectin and laminins, and activates MMP-9 and -13 in addition to MMP-2. MT1-MMP also functions as a collagenolytic growth factor that provides cancer cells with the ability to accelerate proliferative responses by overcoming the growthsuppressive signals from three-dimensional (3D) collagen [9-12]. Inhibition of MT1-MMP was shown to suppress cancer invasion in vitro and in vivo; however, its function in the context of invasion-promoting signals and effector proteins is unclear. A comparative analysis of invasive and noninvasive cells can provide insight into the invasion network of cancer cells. This requires an apparatus that can separate these two cell populations while they are in the active state.

Many approaches have been used to date to analyze cell invasion, including the Transwell invasion (Boyden chamber), 3D cell tracking, vertical gel invasion, spheroid gel invasion and microcarrier bead cell invasion assays [13-19]. The
Transwell invasion assay with a Boyden chamber is the most frequently used of these approaches $[13,16]$. In this method, cancer cells invade through a thin layer of ECM overlying a porous membrane in the chamber and adhere to the bottom of the membrane. Therefore, invasive cells cannot be isolated as they are in the layer of ECM.

This study describes a modified invasion assay in 3D collagen gel using an easy and low-cost apparatus that enables invasive and noninvasive living cells to be separately isolated in their native state from collagen.

\section{Materials \& methods}

\section{Cell culture \& reagents}

HT1080 human fibrosarcoma and HeLa cells were obtained from the American Type Culture Collection (VA, USA) and were maintained in Dulbecco's modified Eagle's medium (DMEM; Sigma-Aldrich, MO, USA) supplemented with $10 \%$ fetal bovine serum. Cellmatrix Type IA (acetic acidextracted type I collagen from pig tendon)

\section{METHOD SUMMARY}

Two collagen gel layers were formed across the membrane of a cell strainer, with only the lower layer containing cells. Cell movement was directed upward. After incubation, gel layers were separated and invading cells in the upper gel could be observed under a light microscope without staining. 
was purchased from Nitta Gelatin (Osaka, Japan). The synthetic MMP inhibitor BB94 was a gift from Kotobuki Pharmaceutical Co. (Nagano, Japan) and was dissolved in dimethyl sulfoxide (DMSO). Anti-FLAG and -tubulin antibodies were purchased from Sigma-Aldrich. Hoechst 33342 was from Molecular Probes (OR, USA). Collagenase I was obtained from Nacalai Tesque (Kyoto, Japan).
Invading cell trapping (iCT) assay

Type I collagen gel ( $3 \mathrm{mg} / \mathrm{ml})$ was diluted to $2.1 \mathrm{mg} / \mathrm{ml}$ by adding $5 \times$ DMEM and $10 \times$ reconstitution buffer $(200 \mathrm{mM}$ HEPES, $260 \mathrm{mM} \mathrm{NaHCO}$, and $50 \mathrm{mM} \mathrm{NaOH}$ ) according to the manufacturer's instructions (Nitta Gelatin). Cell strainers with a nylon mesh of $40-\mu \mathrm{m}^{2}$ square pores (Funakoshi, Tokyo, Japan; Figure 1A \& B) were selected for the iCT assay. A 200- $\mu$ l volume of the
(A)

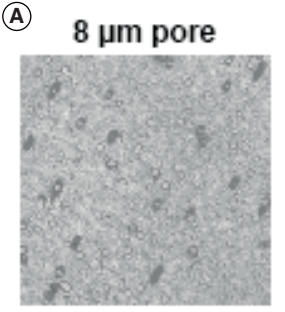

$40 \mu \mathrm{m}$ nylon mesh

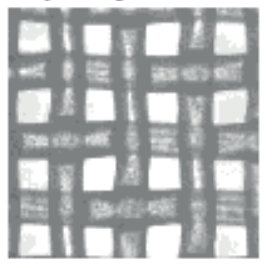

(B) Cells in Collagen gel
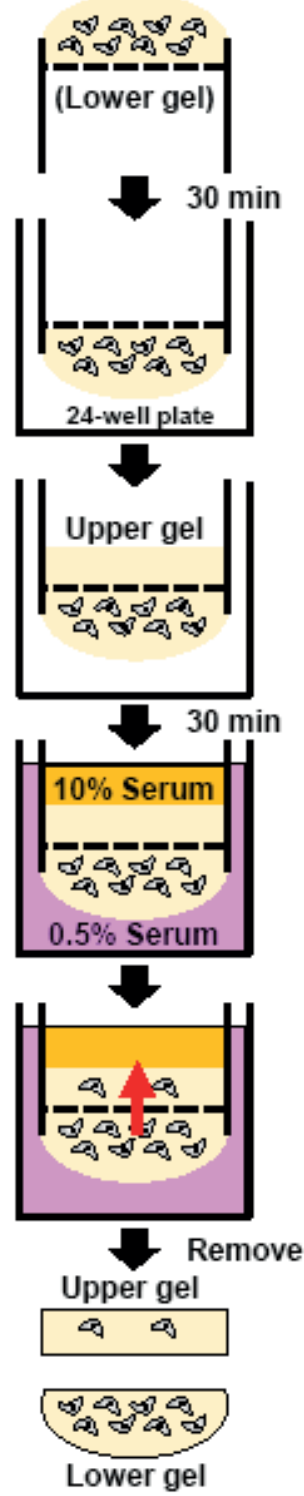

(C)
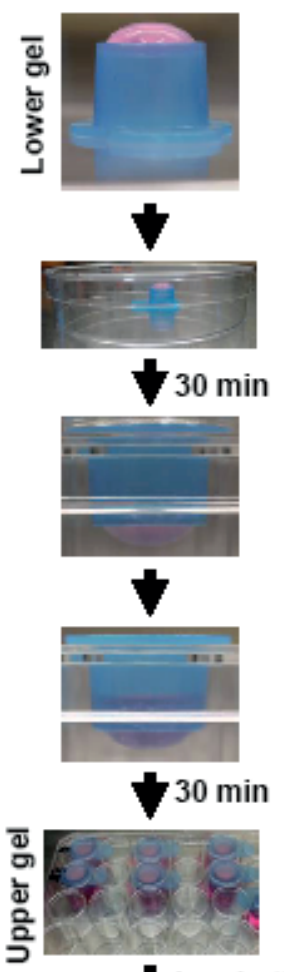

Incubation
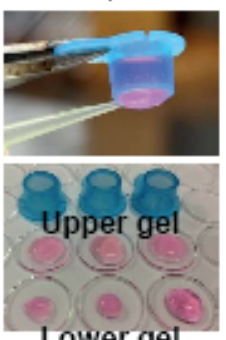

Lower gel

Figure 1. Schematic illustration of the iCT assay. (A) Comparison of polycarbonate membrane with 8- $\mu \mathrm{m}$ pores used for the Boyden chamber assay (Corning Costar, NY, USA) and nylon membrane used for iCT assay with a $40-\mu \mathrm{m}^{2}$ mesh. (B) Concept of the iCT assay. (C) Method of the iCT assay. The cell-collagen mixture was loaded on the bottom surface of the inverted cell strainer. After polymerization, the strainer was returned to its original position and the collagen gel solution was poured into the upper chamber and polymerized. The strainer was placed in a 24 -well plate filled with DMEM containing $0.5 \%$ serum. The inner chamber was filled with DMEM containing $10 \%$ serum to attract cells in the lower gel. After incubation at $37^{\circ} \mathrm{C}$, both upper and lower gels were removed from the strainer and analyzed under a light microscope. iCT: Invading cell trapping.

collagen gel mixture containing test cells was polymerized on the outer surface of the nylon mesh of the cell strainer (Figure 1B), by incubating in a 100-mm diameter culture dish at $37^{\circ} \mathrm{C}$ for $30 \mathrm{~min}$. Nylon mesh with a larger pore size was unable to retain a drop of collagen gel mixture by surface tension, and could not be used. After polymerization, the cell strainer was transferred to a 24-well plate, and $200 \mu \mathrm{l}$ of cell-free collagen gel mixture was poured onto the inner surface of nylon mesh to form an upper gel by incubating at $37^{\circ} \mathrm{C}$ for $30 \mathrm{~min}$. Then, the cell strainer was transferred to a 24-well plate filled with $1700 \mu$ l DMEM containing $0.5 \%$ serum. The inner chamber was filled with $400 \mu$ l of DMEM containing 0.5 or $10 \%$ serum. Cells seeded in the lower gel were allowed to migrate upward into the upper gel for $24 \mathrm{~h}$. Then, the lower and upper gels were separated from the cell strainer with a pipette tip and placed in the 24-well plate. Invaded cells in the upper gel were observed and counted under a light microscope.

\section{Cell viability}

Cell viability was determined by assaying viable cell numbers using a Cell Counting Kit-8 (Dojin Laboratory, Kumamoto, Japan). The living cell number was assessed by measuring the absorbance of $\mathrm{OD}_{490}$ using a SH-1000Lab spectrophotometer (Corona Electric, Ibaraki, Japan). After incubation, the upper and lower gels were removed from cell strainers and incubated in the mixture of $170 \mu$ of DMEM containing $10 \%$ serum, $20 \mu \mathrm{l}$ of Cell Counting Kit-8 reagent, and $10 \mu$ of collagenase at $37^{\circ} \mathrm{C}$ for $4 \mathrm{~h}$. After digesting collagen gels, the relative number of cells in each of the collagen gels were assessed by measuring the absorbance of $\mathrm{OD}_{490}$.

Functional selection of highly invasive cells using the iCT assay To identify highly invasive cells, the iCT assay was carried out using G418-selected HeLa-MT1 cells. After incubation for 3 days, the upper gel containing invading cells was removed and incubated in the 24-well plate for another 6 days. During this period, some cells invaded out of the gel and grew on the surface of the culture plate. Cells in collagen gel were isolated by digesting collagen gel in collagenase I buffer (10\% FBS and $24 \mathrm{U} / \mathrm{ml}$ collagenase I in DMEM) for $1 \mathrm{~h}$ at $37^{\circ} \mathrm{C}$, and were combined with cells invaded out of collagen gel (HeLa-MT1R1 cells). By repeating these steps one more 
time, HeLa-MT1R2 cells were isolated from HeLa-MT1 cells.

Plasmid construction

A cDNA encoding mCherry was PCR amplified from pTRE3G-mCherry (Clontech, Mountain View, CA, USA) and inserted into the pcDNA-FLAG vector at the BamHI and Xbal sites. MT1-MMP cDNA amplified by PCR was then inserted into the pcDNAFLAG-mCherry vector at the HindIII and BamHI sites to generate the pcDNA-MT1MMP-FLAG-mCherry plasmid.

\section{Nuclear staining}

After washing with phosphate-buffered saline (PBS), the gels were fixed with $4 \%$ paraformaldehyde for $4 \mathrm{~h}$, and cells were stained with Hoechst 33342 for 2 h. Fluorescence images were acquired with a confocal laser scanning microscope (LSM510; Carl Zeiss, Jena, Germany). Cells that had invaded into the gel were quantified from these images using ImageJ software (National Institutes of Health, MD, USA).

\section{Gelatin zymography}

Conditioned medium (CM) from the outer chamber was analyzed by gelatin zymography as previously described [6-8]. Briefly, MMP-2 and -9 levels in the supernatant were measured by adding an equal volume of zymography sample buffer. To detect cellbound MMP-2 and -9 , cells embedded in collagen gels were washed twice with PBS and then dissolved in $100 \mu$ l of zymography sample buffer with sonication. The samples were incubated at $37^{\circ} \mathrm{C}$ for $30 \mathrm{~min}$ and then separated by electrophoresis on a sodium dodecyl sulfate (SDS)-polyacrylamide gel containing gelatin labeled with Alexa Fluor 680 (Molecular Probes). Gels were processed and monitored with an Odyssey infrared (IR) imaging system (LI-COR, NE, USA) as previously described.

\section{Western blotting}

After washing twice with PBS, cells were lysed in $10 \mathrm{mM}$ Tris- $\mathrm{HCl}$ (pH 7.5), $100 \mathrm{mM}$ $\mathrm{NaCl}, 5 \mathrm{mM}$ EDTA, $2 \mathrm{mM} \mathrm{Na}_{3} \mathrm{VO}_{4}, 2 \mathrm{mM}$ $\mathrm{NaF}, 1 \%$ SDS, and protease inhibitor cocktail (Nanalai Tesque). Cells in collagen gels were homogenized in SDS-sample buffer. After sonication, the samples were centrifuged to remove cellular debris. Protein concentration was determined with the bicinchoninic acid assay (Pierce, IL, USA). The samples were separated by electrophoresis on SDSpolyacrylamide gels and transferred to a
(A)
(B)
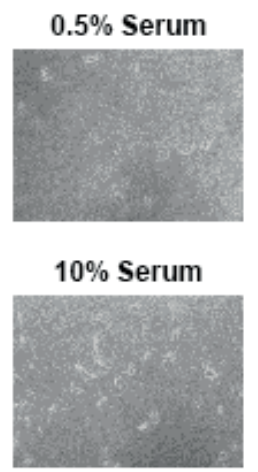

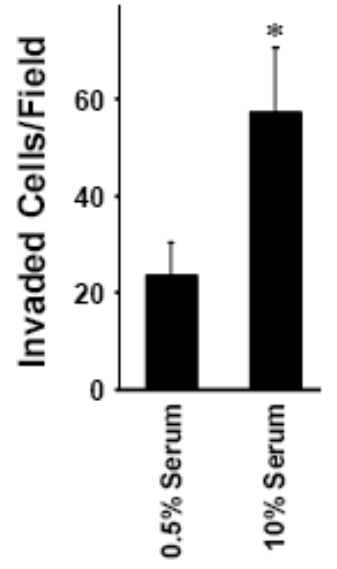

(C)

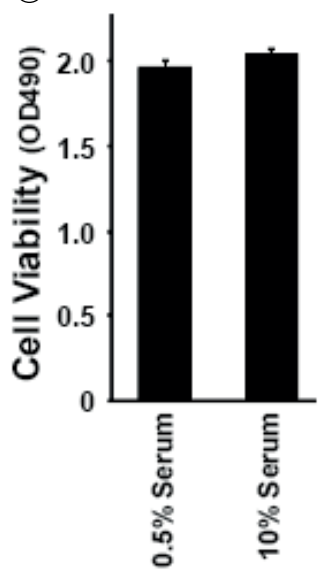

Figure 2. Gel-to-gel invasion of HT1080 cells analyzed with the iCT assay. HT1080 cells $\left(5 \times 10^{5}\right)$ were seeded in the lower gel and invasion into the upper gel was analyzed. Invasion was induced by adding indicated concentrations of serum $(0.5$ or $10 \%)$ to the upper gel layer and incubating for 24 h. (A) Phase contrast images of invading cells in the upper gel. (B) Number of cells in the upper gels was counted under a light microscope. Average number of cells per field is presented. Each condition was run in duplicates, and data represent the means \pm SD of two independent experiments. The asterisk indicates statistical significance $(p<0.05)$. (C) Number of cells in the lower gels was assayed by measuring viable cell numbers using a Cell Counting Kit-8. The living cell number was assessed by measuring the absorbance of $\mathrm{OD}_{490}$ using a SH-1000Lab spectrophotometer. Each condition was run in duplicates, and data represent the means \pm SD of two independent experiments. iCT: Invading cell trapping.
(A)
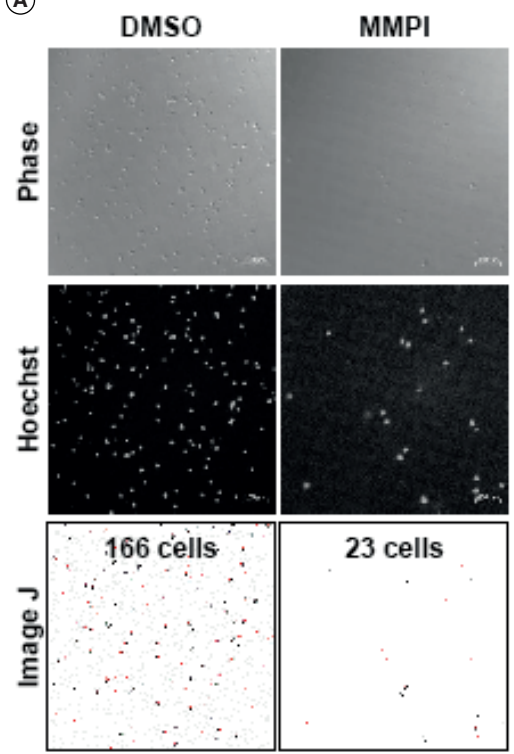

(B)

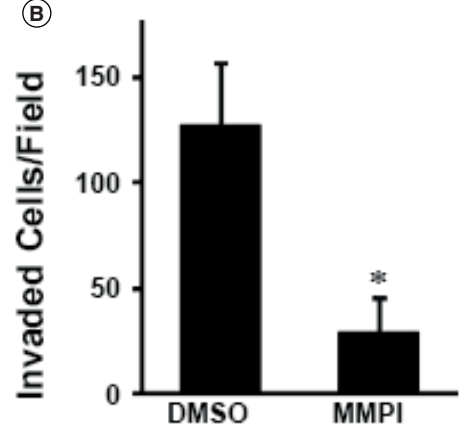

(C)

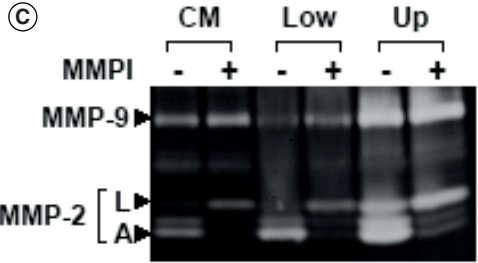

Figure 3. MMP-dependent cell invasion. Invasion of HT1080 cells $\left(2 \times 10^{5}\right.$ cells/chamber) was examined for 3 days in the presence of the MMP inhibitor BB94 $(2 \mu \mathrm{M})$ or solvent (DMSO). (A) Invading cells in the upper gel were stained with Hoechst 33342 (1:5000), and images were obtained with a confocal laser scanning microscope (top and middle panels). The number of invading cells was automatically counted using ImageJ software (bottom panel). Scale bars, $100 \mu \mathrm{m}$. (B) Number of invading cells per field. Each condition was run in duplicates, and data represent the means \pm SD of two independent experiment. The asterisk indicates statistical significance $(p<0.05)$. (C) CM obtained from the outer chamber and cell lysates from the lower (Low) and upper (Up) gels were subjected to gelatin zymography.

A: Active form of MMP-2; CM: Conditioned medium; L: Latent form of MMP-2; MMP: Matrix metalloproteinase. 
nitrocellulose membrane that was probed with the appropriate antibodies. The signal was detected with the Odyssey IR imaging system.

\section{Statistics}

Data were analyzed with the two-tailed Mann-Whitney $U$ test. $p<0.05$ was considered significant.

\section{Results \& discussion}

The iCT assay was developed and used to isolate invasive cells from a 3D collagen matrix. HT1080 human fibrosarcoma cells were used as a highly invasive cell line. Briefly, the apparatus had two collagen gel layers separated by a nylon mesh membrane with $40-\mu \mathrm{m}^{2}$ pores that were large enough to allow passage of invading cells and also facilitated the separation of collagen gel layers at the end of the assay. The layers were formed across the membrane using a cell strainer. The bottom of the strainer harbored a short skirt structure that held the lower gel during the assay. To stimulate cell movement against the force of gravity, cells were seeded in the lower gel and their

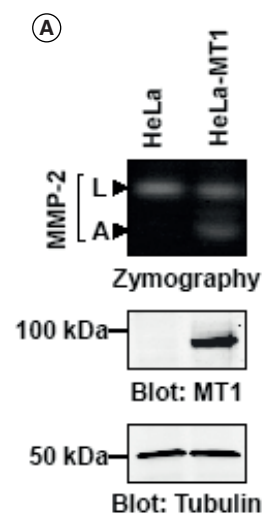

(D)

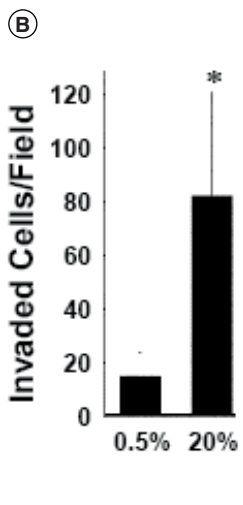

\section{(c)}

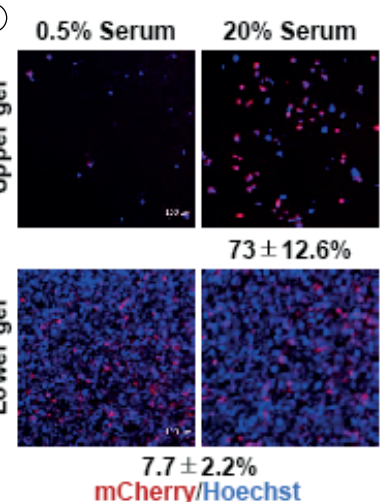

(E)
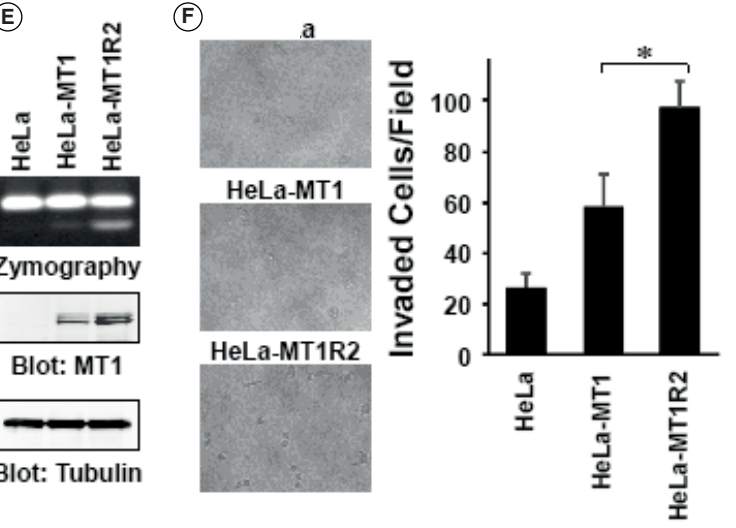

Figure 4. Analysis of MT1-MMP-dependent invasion. HeLa cells were transfected with MT1-MMP-FLAGmCherry plasmid to obtain MT1-mCherry-expressing cells (HeLa-MT1). (A) CM from the cells was analyzed by gelatin zymography. Activities corresponding to $L$ and $A$ forms of MMP-2 are shown (top panel). Cell lysates were analyzed by western blotting using anti-FLAG (MT1; 1:2000, middle panel) and anti-tubulin (1:2000, bottom panel) antibodies. (B) A mixture of parent HeLa cells and HeLa-MT1 cells $\left(4 \times 10^{5} /\right.$ chamber) was evaluated with the iCT assay for 3 days in the presence of 0.5 or $20 \%$ serum. Cells in the upper gel were counted under a light microscope. The number of invading cells per field is presented. Each condition was run in duplicates, and data represent the means \pm SD of two independent experiments. The asterisk indicates statistical significance $(p<0.05)$. (C) Cells in the lower and upper gels were fixed and stained with Hoechst 33342, and images were obtained with a confocal laser scanning microscope. Scale bars, $100 \mu \mathrm{m}$. (D) Alternatively, serum-stimulated cells in the lower and upper gels were analyzed by western blotting using anti-FLAG (MT1) and anti-tubulin antibodies. The MT1-MMP/ tubulin ratio was analyzed using Image J software. (E) CMs from HeLa, HeLa-MT1 and HeLa-MT1R2 cells were analyzed by gelatin zymography (top panel). Cell lysates were analyzed by western blotting using anti-FLAG (MT1) (middle panel) and anti-tubulin (bottom panel) antibodies. (F) HeLa, HeLa-MT1 and HeLa-MT1R2 cells $\left(3 \times 10^{5} /\right.$ chamber) were evaluated with the iCT assay for 3 days in the presence of $20 \%$ serum. Cells in the upper gel were counted under a light microscope (right panel). The number of invading cells per field is presented. Each condition was run in duplicates, and data represent the means \pm SD of two independent experiments. The asterisk indicates statistical significance $(p<0.05)$. A: Active form of MMP-2; CM: Conditioned medium; L: Latent form of MMP-2; MMP: Matrix metalloproteinase. movement was directed upward (Figure 1B). The downward migration of cells seeded in the upper gel can also be examined using this apparatus.

The cells were mixed with neutralized type I collagen and placed on the top surface of the inverted strainer (corresponding to the lower gel; Figure 1C). After polymerization of the cell-collagen mixture, the chamber was placed in a 24-well plate, and another collagen gel was cast inside the strainer (upper gel). Medium containing 0.5\% serum was added to each well of the plate and medium containing 10\% serum was added to the upper gel in the strainer. Cells in the apparatus were allowed to migrate into the collagen gel across the membrane mesh. After incubation for $24 \mathrm{~h}$, the cell strainer was removed from the plate and gel layers were separately removed. Noninvading cells remained in the lower gel, whereas invading cells were recovered from the upper collagen gel and counted under a light microscope (Figure 2A). Cell number in the upper gel under $10 \%$ serum condition was significantly larger than those of $0.5 \%$ serum culture (Figure 2B). However, cell number in the lower gel was almost identical between 0.5 and $10 \%$ serum condition (Figure $2 \mathrm{C}$ ). These results suggest that the iCT assay enables the analysis of invasion stimulated by a higher serum concentration.

HT1080 cells express multiple MMPs including MT1-MMP and MMP-2 and -9, and it was previously reported that MMP activity determines the ability of cells to invade collagen $[7,8]$. To confirm whether the observed invasion was dependent on MMP, the assay was carried out in the presence of the synthetic MMP inhibitor (MMPI) BB94. We found that HT1080 cell invasion was suppressed by MMPI (Figure 3A \& B), while cell number both in the lower gel and upper gel was unaffected by MMPI treatment (Supplementary Figure 1). The upper gel was fixed and stained with Hoechst 33342 and examined by confocal laser scanning microscopy (Figure 3B); a quantitative analysis of invading cells confirmed that MMPI treatment reduced cell invasion.

Gelatin zymography allows visualization of gelatinolytic activity in samples. Latent and active forms of MMP-2 and -9 exhibit potent gelatinase activity in gelatin zymography. MT1-MMP activity can be evaluated based on the presence of the active form of MMP-2. CM and lysates of cells in the upper and lower gels were analyzed by zymography to detect MMP-2 
and -9 produced by HT1080 cells (Figure 3C). Gelatinolytic activities associated with MMP-2 and -9 were observed in CM and cell lysates. The active form of MMP-2 was also observed, but this was decreased by MMPI treatment, indicating that inhibition of MT1-MMP suppressed activation of latent MMP-2. These results demonstrate that the iCT assay reflects cells' ability to invade the collagen gel in an MMP-dependent manner, consistent with earlier observations [6-12].

To confirm the effect of MT1-MMP on cell invasion, HeLa cells were transfected with a plasmid expressing FLAG and mCherry-tagged MT1-MMP. HeLa-MT1 cells were obtained by G418 selection for 3 weeks, which expressed MT1-FLAGmCherry and thereby activated MMP-2 (Figure 4A). HeLa-MT1 cells were mixed with the parental HeLa cells and analyzed with the iCT assay; $7.7 \%$ of cells in the mixture were mCherry-positive. After incubation for 3 days, the upper gel was removed and the number of mCherry-positive cells was evaluated by confocal microscopy. The number of invading cells was fivefold higher in the presence of $20 \%$ as compared with $0.5 \%$ serum (Figure 4B). Accordingly, the fraction of mCherry-positive cells in the upper gel was increased to 73\% (Figure 4C). Western blotting of the cells embedded in the collagen gel showed that the MT1-MMP/ tubulin ratio was 7.5-fold higher in the cells that had invaded the upper gel than in the cells that stayed in the lower gel (Figure 4D). These results indicate that MT1-MMPexpressing HeLa cells were more invasive than the parent cells and were enriched in the upper gel.

To test whether highly invasive cells could be selected by repeating the $\mathrm{iCT}$ assay, we performed the iCT assay using HeLa-MT1 cells. After incubation, the invading cells embedded in the upper gel were cultured for another 6 days. After degrading the collagen gel using collagenase, the cells grew in the culture dish (HeLa-MT1R1 cells). By repeating this protocol using HeLa-MT1R1 cells, we obtained HeLa-MT1R2 cells. These HeLa-MT1R2 cells expressed higher levels of MT1-MMP and activated MMP-2 more effectively than the parent HeLa and HeLa-MT1 cells (Figure 4E). Thus, HeLa-MT1R2 cells invaded into the upper gel more efficiently than HeLa and HeLa-MT1 cells (Figure 4F). These results indicate that the iCT assay could be used to select the cells that are functionally more invasive from parent cells.
Collagen is a major component of tissue surrounding cancer cells, representing approximately $30 \%$ of total protein mass. Type I collagen is the main scaffold protein in the stromal ECM $[20,21]$. Invasion of the collagen matrix was shown to be correlated with invasive and metastatic capacity of cancer cells. Since enrichment of invasive cells in their active state enables analysis of the molecular mechanisms involved, we developed a simple and low-cost method for this purpose. Using the iCT assay we confirmed that MT1-MMP plays a crucial role in cancer cell invasion into the collagen gel (Figures 2-4). The advantage of the iCT assay is that it captures invasive and noninvasive cells in two separate collagen gel layers; a nylon mesh membrane between the layers allows easy removal of the gels from the apparatus and means that there is no need for a specialized microscope and imaging analysis software. Invading cells in the upper gel can be examined under a light microscope. The viability of both invaded and noninvaded cells remaining in the gels can be examined by typical colorimetric assays, if desired. By repeating the $\mathrm{iCT}$ assay, highly invasive HeLa-MT1R2 cells, which expressed higher levels of MT1-MMP and activated MMP-2 more effectively than the parent HeLa and HeLa-MT1 cells, were isolated (Figure 4). There are currently no other assays that can selectively and efficiently trap invading cells within a 3D microenvironment.

The iCT assay is similar to other methods in that it is also an inverted invasion assay $[18,19]$. The advantage of the iCT assay is that it allows the two collagen gel layers to be easily separated, with living invaded and noninvaded cells trapped in the upper and lower gels, respectively. We monitored the upward invasion of cancer cells in this study; however, cells can also be embedded in the upper gel using this device, in which case downward invasion would be monitored. There are also disadvantages. The excessive collagenolysis caused by a large number of cells and a long incubation period failed to induce upward invasion. In such a case, monitoring downward invasion by seeding cells in the upper gel might be appropriate. The collagen gel does not fully mimic the in vivo microenvironment in cancer cell invasion. Adding other ECM components such as laminins and fibronectins to the collagen gel [19], as well as growth factors and cytokines to the medium, may better recapitulate the in vivo tumor microenvironment.

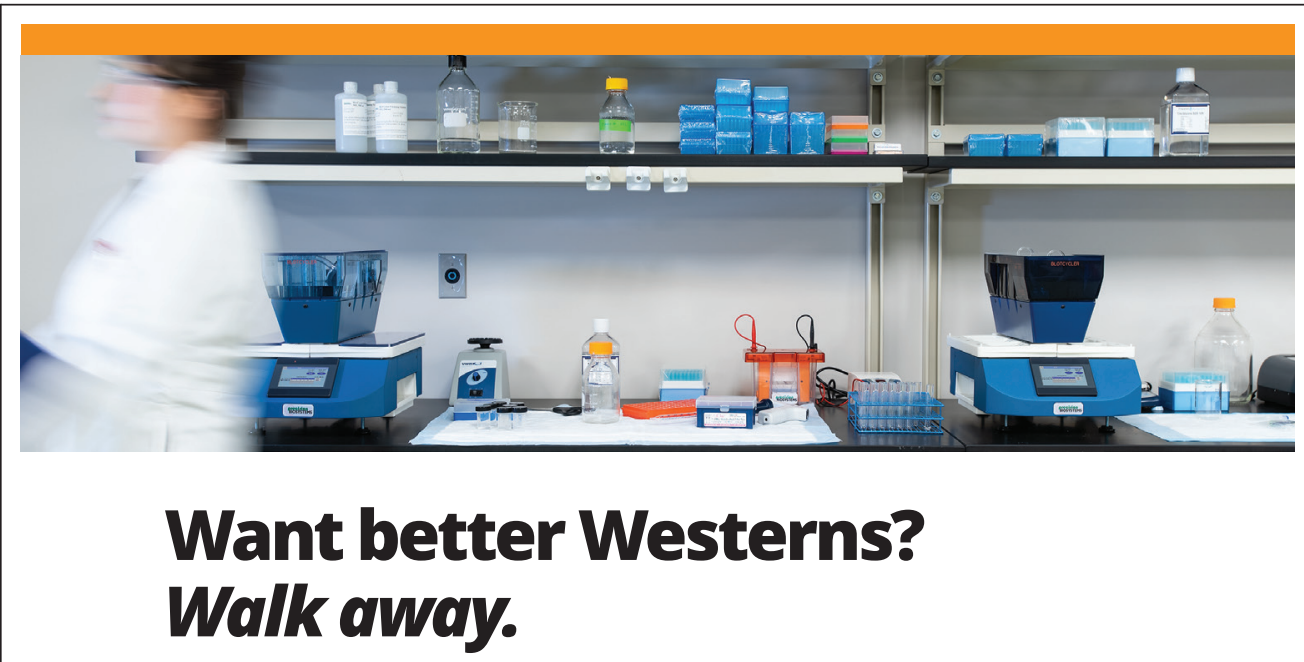

Traditional western blot analysis is a time-consuming process. BlotCycler $^{\text {TM }}$ changes everything by automating the fluid changes and timing associated with blocking, washing and antibody

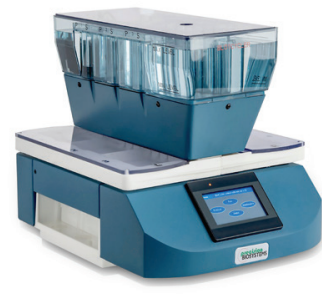
incubation. There's no more need to watch over the procedure. With BlotCycler, simply set up your protocol, and walk away!

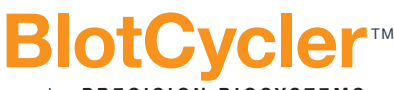

www.blotcycler.com 
Invasive and noninvasive cell populations can be further enriched by repeating the iCT assay and may be used for transcriptome, proteome and genome analyses.

\section{Author contributions}

TT contributed to experimental and assay design, performed experiments, and wrote the first draft of the manuscript. HS and MS supervised the study, contributed to the experimental design, and edited the manuscript.

\section{Financial \& competing interests disclosure}

This work was supported by JSPS KAKENHI Grant Number JP16H04695 to M Seiki. The authors have no other relevant affiliations or financial involvement with any organization or entity with a financial interest in or financial conflict with the subject matter or materials discussed in the manuscript apart from those disclosed.

No writing assistance was utilized in the production of this manuscript.

\section{Acknowledgements}

We would like to thank Editage (www. editage.jp) for English language editing.

\section{Open access}

This work is licensed under the Creative Commons Attribution 4.0 License. To view a copy of this license, visit http:// creativecommons.org/licenses/by/4.0/

\section{Supplementary data}

To view the supplementary data that accompany this paper please visit the journal website at: https://www.future-science.com/ doi/suppl/10.2144/btn-2018-0036

\section{References}

1. Bonnans C, Chou J, Werb Z. Remodelling the extracellular matrix in development and disease. Nat. Rev. Mol. Cell Biol. 15, 786-801 (2014).

2. Larsen M, Artym VV, Green JA, Yamada KM The matrix reorganized: extracellular matrix remodeling and integrin signaling. Curr. Opin. Cell Biol. 18, 463-471 (2006).

3. Sato $H$, Takino T, Miyamori H. Roles of membrane-type matrix metalloproteinase-1 in tumor invasion and metastasis. Cancer Sci. 96, 212-217 (2005)

4. Itoh Y, Seiki M. MT1-MMP: a potent modifier of pericellular microenvironment. J. Cell. Physiol. 20, 1-8 (2006). 5. Wolf K, Friedl P. Extracellular matrix determinants of proteolytic and non-proteolytic cell migration. Trends Cell. Biol. 21, 736-744 (2011).

6. Sato $\mathrm{H}$, Takino $\mathrm{T}$, Okada $\mathrm{Y}$ et al. A matrix metalloproteinase expressed on the surface of invasive tumour cells. Nature 370, 61-65 (1994)

7. Takino $\mathrm{T}$, Miyamori $\mathrm{H}$, Watanabe $Y$, Yoshioka K, Seiki M, Sato H. Membrane type 1 matrix metalloproteinase regulates collagen-dependent mitogen-activated protein/extracellular signal-related kinase activation and cell migration. Cancer Res. 64, 1044-1049 (2004).

8. Takino T, Saeki H, Miyamori $\mathrm{H}$, Kudo T, Sato H. Inhibition of membrane-type 1 matrix metalloproteinase at cell-matrix adhesions. Cancer Res. 67, 11621-11629 (2007).

9. Holmbeck K, Bianco P, Caterina $\mathrm{J}$ et al. MT1-MMPdeficient mice develop dwarfism, osteopenia, arthritis, and connective tissue disease due to inadequate collagen turnover. Cell 99, 81-92 (1999). 10. Hotary K, Allen E, Punturieri A, Yana I, Weiss SJ. Regulation of cell invasion and morphogenesis in a threedimensional type I collagen matrix by membranetype matrix metalloproteinases 1, 2, and 3. J. Cell Biol. 149, 1309-1323 (2000).

11. Hotary KB, Allen ED, Brooks PC, Datta NS, Long MW, Weiss SJ. Membrane type I matrix metalloproteinase usurps tumor growth control imposed by the three-dimensional extracellular matrix. Cell 114, 33-45 (2003).

12. Takino T, Tsuge H, Ozawa T, Sato H. MT1-MMP promotes cell growth and ERK activation through c-Src and paxillin in three-dimensional collagen matrix. Biochem. Biophys. Res. Commun. 396, 1042-1047 (2010).

13. Albini $A$, Iwamoto $Y$, Kleinman $\mathrm{HK}$ et al. A rapid in vitro assay for quantitating the invasive potential of tumor cells. Cancer Res. 47, 3239 3245 (1987).

14. Wolf $\mathrm{K}$, Mazo I, Leung $\mathrm{H}$ et al. Compensation mechanism in tumor cell migration: mesenchymal-amoeboid transition after blocking of pericellular proteolysis. J. Cell Biol. 160, $267-$ 277 (2003)

15. Park DW, Choi DS, Ryu HS, Kwon HC, Joo $\mathrm{H}$, Min CK. A well-defined in vitro threedimensional culture of human endometrium and its applicability to endometrial cancer invasion. Cancer Lett. 195, 185-192 (2003).

16. Kramer NA, Walzl C, Unger M et al. In vitro cell migration and invasion assays. Mutat. Res. 752 10-24 (2013).

17. Miller MC, Manning HB, Jain A et al. Membrane type 1 matrix metalloproteinase is a crucial promoter of synovial invasion in human rheumatoid arthritis. Arthritis Rheum. 60, 686697 (2009)

18. MacArdle TJ, Ogle BM, Noubissi FK. An in vitro inverted vertical invasion assay to avoid manipulation of rare or sensitive cell types. J. Cancer 7, 2333-2340 (2016)

19. Brekhman V, Neufeld G. A novel asymmetric 3D in-vitro assay for the study of tumor cell invasion. BMC Cancer 9, 415 (2009).

20. Egeblad M, Rasch MG, Weaver VN. Dynamic interplay between the collagen scaffold and tumor evolution. Curr. Opin. Cell Biol. 22, 697706 (2010)

21. Shields MA, Dangi-Garimella S, Redig AJ, Munshi HG. Biochemical role of the collagen-rich tumour microenvironment in pancreatic cancer progression. Biochem. J. 441, 541-552 (2012).

First draft submitted: 28 November 2017; Accepted for publication: 15 June 2018

Address correspondence to: Takahisa Takino; Division of Education for Global Standard, Institute of Liberal Arts \& Science, Kanazawa University, Kakuma-machi, Kanazawa 920-1192, Japan; Tel.: +81 76264 6710; Fax: +81 76234 4505; E-mail: ttakino@staff.kanazawa-u.ac.jp

To purchase reprints of this article contact: s.cavana@future-science.com
Broadest Fluorophore Cov
DAPI-Cy7 and beyond...

\section{Enhanced Intensity}

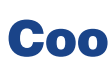

Simply Better Control

For more information:

+44 (0)1264323040 (Worldwide) 1-800-877-0128 (USA/Canada) e: info@CoolLED.com 